\title{
REFLEXIONES SOBRE EL TRABAJO SANITARIO CON PERSONAS MAYORES EN LA ADMINISTRACIÓN PÚBLICA. ESTUDIO DE CASOS
}

\author{
REFLECTIONS ON HEALTH STAFF WORKING WITH ELDERLY PEOPLE \\ IN THE PUBLIC ADMINISTRATION. CASE STUDIES
}

\author{
Concepción Nieto-Morales \\ Universidad Pablo de Olavide, Sevilla. España/Spain \\ concepcionm@upo.es \\ Lourdes Nieto-Cabrera \\ Klinikum Darmstadt. Alemania/Germany \\ lourdes-nieto@hotmail.com \\ Manuel Francisco Díaz González \\ Universidad Pablo de Olavide, Sevilla. España/Spain \\ mdiagon@upo.es
}

Recibido/Received: 08/12/2012

Modificado/Modified: 15/04/2013

Aceptado/Accepted: 09/09/2013

\section{RESUMEN}

En este trabajo se analiza el discurso de profesionales sanitarias vocacionales que trabajan en residencias públicas, desde una perspectiva de empleo público y cuidados personales. La crisis ha destruido empleos, derechos sociales, ha precarizado el trabajo que se realiza en todos los ámbitos y de forma especial en la Administración, se comienza a externalizar y subcontratar el trabajo, a flexibilizar las condiciones laborales, se crea inestabilidad lo que conlleva empeorar las condiciones de los profesionales públicos y sanitarios en particular. Hay que preguntarse dónde quedan la estabilidad laboral, la vocación y trabajar en lo que cada persona se ha especializado. Cada vez más se empeoran las condiciones laborales, produciéndose gran precariedad laboral, las estadísticas reflejan que a más formación menor desempleo, pero también es cierto que existen muchos universitarios trabajando en sectores diferentes a los que se han formado. Para centrarse en este trabajo se ha realizado un grupo de discusión compuesto por 6 personas, Diplomadas Universitarias en Enfermería y Auxiliares de Enfermería que trabajan en residencias públicas para personas mayores; dicho grupo de discusión se realiza fuera del centro laboral.

\section{PALABRAS CLAVE}

Empresa, empleo, inestabilidad, flexibilidad, precariedad.

\section{SUMARIO}

1. Introducción. 2. Empresa, Trabajo, Empleo. 3. Análisis de discursos de profesionales sanitarios. 4. A modo de conclusión. Bibliografía.

\section{ABSTRACT}

The speech of female health professionals who work in public residences is analyzed in this writing, a work which most of them choose as their vocation, from a perspective in public employment and personal cares. The crisis has destroyed positions, social rights, made precarious the employment done 
in all the environments and especially in the Administration, the outsourcing of work is started, the increasing of flexibility of the labor conditions, the instability... Everything worsens particularly the conditions of the public health professionals. The question arisen is: where are the labor stability, the vocation and working in a position each one has been trained for? The labor conditions worsen, making employment and positions more precarious. The statistics reflect there is less unemployment when there is more training; but there are many university people who work in a position different to the one they have been trained for. A group of discussion composed by six people with a university degree in Nursing and Auxiliary Nursing Care, who work in public residences for elderly people, has been created to focus on this work, out of the labor place.

\section{KEYWORDS}

Company, employment, instability, flexibility, precariousness.

\section{CONTENTS}

1. Introduction. 2. Company, work, employment. 3. Analysis of health professionals' speech. 4. To conclude. References.

\section{INTRODUCCIÓN}

El trabajo es uno de los ejes sobre los que pivota la vida de las personas en las sociedades avanzadas y al que destinamos gran parte del tiempo que además nos permite la inclusión social y proporciona los ingresos para poder tener una vida normalizada.

En el último siglo y medio, los progresos sociales y laborales han experimentado un gran avance, que se ha visto frenado con la grave crisis que nos aqueja en los últimos años y de la que no se vislumbra el final del camino. Los cambios económicos e ideológicos, han desencadenado legislaciones que flexibilizan el mercado laboral y deteriora de forma importante y hace más deficitaria las condiciones de los empleados.

Con los cambios sociales que se estaban produciendo desde hace al menos dos décadas se comenzó a implantar la formación a lo largo de la vida, dado que las previsiones eran que se cambiara de trabajo $\mathrm{u}$ oficio en el transcurso de la vida laboral varias veces, debiendo el trabajador adaptarse a las nuevas realidades.

Desde mediados del siglo XIX y durante todo el siglo XX los importantes logros a nivel laboral en beneficio de los trabajadores, que con la reforma de las últimas legislaciones laborales se han destruido, precarizan el mercado y produce efecto dominó a nivel económico y social, repercutiendo gravemente en las personas.

Desde los años 80 del siglo XX se comenzó a externalizar y subcontratar el trabajo, a flexibilizar las condiciones laborales, con la excusa de que eran mas rentables, aunque el análisis de este tema es verdaderamente complejo y requeriría un estudio en profundidad, porque si esto es verdaderamente como se presentaba, dado que si se externaliza el trabajo a una empresa privada los propietarios de la empresa indudablemente deben obtener beneficios, que por otra parte no los obtiene la empresa pública, por lo que hay que preguntarse dónde están los tan aireados beneficios de quienes exponen dichos criterios, porque socialmente no se perciben, dado que los trabajadores tienen peores condiciones laborales y perciben menores emolumentos y el coste para la Administración es el mismo importe. Esta cuestión se produce en centros residenciales de la Junta de Andalucía. 
En los últimos tiempos lo que impera es desempleo masivo y gran precariedad laboral, las estadísticas reflejan que a más formación menor desempleo, pero también es cierto que existen muchos universitarios trabajando en sectores diferentes a los que se han formado.

Ante tal panorama hay que preguntarse qué estabilidad laboral tendrán las generaciones futuras y si será posible trabajar en lo que cada persona se ha especializado.

Para este artículo se ha realizado un grupo de discusión que en principio se había previsto realizar con 7 personas de las cuales 6 mujeres y un hombre pero en el último momento por motivos de enfermedad el hombre no acudió y se optó por realizarlo con las 6 personas Diplomados Universitarios en Enfermería y auxiliares de enfermería que trabajan en residencias públicas, que facilitan información sobre sus percepciones sobre el trabajo y el mundo laboral.

\section{EMPRESA, TRABAJO, EMPLEO.}

Tener trabajo, percibir un sueldo por él es una cuestión fundamental en la vida para la inclusión social, no es cuestión baladí y se recoge en la Constitución (1978) española.

"El trabajo, derecho y deber": "1. Todos los españoles tienen el deber de trabajar y el derecho al trabajo, a la libre elección de profesión u oficio, a la promoción a través del trabajo y a una remuneración suficiente para satisfacer sus necesidades y las de su familia, sin que en ningún caso pueda hacerse discriminación por razón de sexo. 2. La ley regulará un estatuto de los trabajadores" (art. 35).

La actual sociedad y modo de producción capitalista, presenta condiciones de vida y trabajo con nuevas formas de producción que escapan al control de los Estados y buena prueba de ello se encuentra en las crisis económicas cíclicas que se producen y que escapa a control de los gobiernos de cualquier país.

Hay que diferenciar entre la empresa privada que persigue unos beneficios y produce crecimiento y riqueza y la empresa publica en las sociedades democráticas destinadas a prestar un servicio publico.

La cultura empresarial de cualquier empresa es única y se basa en las formas de gestionar sus pilares básicos determinado por valores, normas, principios, hábitos, conocimientos, comunicación, comportamientos, etc. (Sánchez Herrera y Pintado Blanco, 2009). Los nuevos tiempos obligan a redirigir los esfuerzos e identificar las oportunidades que se presentan.

Desde el último cuarto de siglo XX, se produjeron cambios en las empresas a todos los niveles, desde la gestión, dirección, recursos humanos (RRHH), etc.; dichos cambios modifican las formas de empleo hasta el momento continuo y prolongado a lo largo de la vida con carrera profesional (Zubero, 2006:18). El trabajo hay que diferenciarlo del empleo, el empleo se encuentra ligado a una actividad productiva al tiempo que el trabajo se encuentra desligado de una actividad profesional (Castillo Mendoza; García López, 2001).

Las normas para acceder al empleo publico son especificas debiendo tener difusión y accesibilidad a todos los ciudadanos y ser publicadas en el boletín oficial del gobierno, ya sea Estatal, Autonómico, Local, etc. Para los empleados públicos que acceden a un puesto de trabajo en la Administración por oposición, se puede garantizar que quien accede a la plaza concurre por méritos, pero no todos los que tienen méritos acceden, dado que existe una selección de los mejores, al menos esta es la consigna, aunque en los últimos tiempos existen resquicios legales para incluir en la nomina de la Administración Pública infiltrados sin dichos méritos. Con la actual crisis, la Central Sindical Independiente y de Funcionarios 
(CSI-F), anuncia que la sanidad en la Administración pública acumula la misma destrucción de empleo que otros sectores como construcción e industria (CSI-F, 2013).

"La empresa privada es el modo de generar crecimiento y riqueza, siendo el empresario un benefactor social que además de generar tales atributos, permite el empleo y la realización material del ser humano trabajador. A este fin, el sector público se descompone, entregándose por los poderes del Estado a la gestión privada, después de predicar la eficiencia de esta frente a la incompetencia de lo público poniéndose en cuestión la necesidad y eficacia de la función pública que al socaire de la relativa mayor estabilidad en el empleo puede ser objeto de reducciones y verdaderas agresiones sobre organismos y personas en sus condiciones de trabajo y retribución; argumentación burdamente ideológica que ignora tanto el acceso a la función pública, como sus prestaciones productivas y de servicios" (Gasco García, 2011:174).

La crisis actual, esta sirviendo de parapeto en algunas Administraciones para privatizar servicios que deben ser prestados de forma publica, cuando el problema se encuentra en la pésima gestión, en parte porque los nombramientos de gestión de niveles administrativos son de libre designación política, situando en cargos a personas poco adecuadas para ciertos puestos.

La educación es fundamental para que todo el mundo tenga las mismas oportunidades, debiendo reflexionar respecto al sistema educativo, el mercado de trabajo y la economía real. El rendimiento académico va a repercutir en el futuro educativo y tendrá efectos ulteriores en el acceso a los estudios que se realicen. Es necesario adaptar la enseñanza a la nueva realidad laboral.

El trabajo que se desempeñará a lo largo de la vida laboral, está directamente relacionado con la formación que se ha desarrollado en la formación reglada; en España según los niveles PISA (2009) no arrojan buenos resultados comparados con otros países europeos; se considera la Formación Profesional (FP) como el punto débil del sistema educativo frente a otros países donde son muy valorados con gran repercusión social e impacto económico (Lavía, et al, 2012). No cabe duda que tras la formación debe concurrir el empleo, para lo cual comienza una etapa administrativa compleja dada la configuración del trabajo en la sociedad actual y la competitividad y estrategia de las empresas en su gestión debiendo priorizar en el desarrollo de los RRHH que son el gran valor activo de las mismas. Si esto es fundamental, se debería de dar relevancia a la relación de puestos de trabajo (RPT), descripción de dichos puestos, políticas saláriales, evaluación de los puestos, competencias tanto personales como laborales en el desempeño profesional, etc.; siendo importante incentivar a quienes realicen un buen trabajo y sean resolutivos; también es importante la percepción, motivación, etc., que los empleados tengan porque la cuestión es que tanto empleador como empleados perciban compensaciones y remen en la misma dirección para que exista éxito empresarial y profesional.

La importancia y desarrollo de los RRHH tuvo gran auge a partir de los años 90 del siglo $\mathrm{XX}$, definiéndose como elementos desde donde la empresa dirige su potencial humano a través de la política empresarial, la estrategia y las practicas, existiendo practicas desde los RRHH enfocadas a la cultura, valores, comunicación, evaluación del desempeño y rendimiento, clima, motivación, equipos de trabajo, carrera profesional, etc., (Boada-Grau, et al, 2012). Hay que individualizar, pero esta claro que el interés general debe estar por encima del particular de cada persona.

Por otra parte el sector sanitario que se ha dicho anteriormente que se encuentra formado mayoritariamente por personal femenino y que representan una mínima parte de las mujeres 
que escapan de empleos generalmente precarios en función del genero y el desencadenante de la feminización de la pobreza existiendo un amplio espectro entre la pobreza femenina y masculina (Belzunegui y Pastor, 2011).

El tiempo que se dedica a la actividad laboral es aproximadamente de un tercio del total, por lo que puede calificarse de importante en la vida de las personas, considerándose que en el trabajo se pasa buena parte de la vida para obtener ingresos que permitan cubrir las necesidades, aunque también es de suma importancia la realización personal y el desarrollo, que desencadenara satisfacción personal y por ende contribuirá a mejor calidad de vida; si no fuera así, se podría convertir en una actividad fatigosa, rutinaria, que hará mella y producirá problemas emocionales en la salud a medio y largo plazo (Alves Corrêa, et al, 2013)

\section{ANÁLISIS DE DISCURSOS DE PROFESIONALES SANITARIOS.}

La profesión sanitaria, formada por Diplomados Universitarios en Enfermería (DUE), Auxiliares de Enfermería, ha estado formada mayoritariamente por mujeres y el personal sanitario o celadores por hombres, aunque desde hace unos años las tendencias están cambiando pero aun persiste, el $84 \%$ de los colegiados de enfermería son mujeres (INE, 2013).

En el grupo de discusión han participado 6 profesionales sanitarias que aunque todas han sido auxiliares de enfermería en el momento de realizarse el grupo de discusión dos de ellas han promocionado a enfermeras y otras dos que también poseen estudios universitarios de Trabajo social y psicología no han podido promocionar por motivos diversos, siguiendo desempeñando el trabajo de auxiliar de enfermería. Los años que llevan trabajando en la Administración en la rama sanitaria oscila entre los 20 y 31 años, y las edades entre los 40 y 51 años, algunas comenzaron a trabajar muy jóvenes, al poco de cumplir la mayoría de edad, cuestión que si comparamos con el momento presente es casi imposible debido a las circunstancias de crisis y la situación de desempleo actual. Se podría pensar que son profesionales privilegiadas respecto a su carrera profesional, pudiendo cambiar de trabajo y teniendo estabilidad laboral, lo que te permite tener otra visión de futuro.

La discriminación entre los colectivos y diferencias en los grupos de trabajo, los superiores trabajan a otro nivel, enrareciendo el ambiente cuanto más tiempo pasa aumenta la frustración personal y en el trabajo, porque no son valorados los trabajos que se realizan, ni los esfuerzos que realizan los trabajadores para que el trabajo salga adelante sin recompensa de ningún tipo, lo que conlleva machacar a la persona, unido a que el trabajo en sí es difícil y desagradable, personas mayores, en estado terminal, unido a mucho trabajo físico y de movilización de pesos muertos, siendo un ambiente triste y viciado (Aux. enfermería 1).

La evolución del trabajo sanitario con personas mayores no es visto por el personal sanitario como satisfactorio porque a las personas mayores dependientes se van deteriorando, no suelen mejorar, al contrario se deterioran a medida que trascurre el paso del tiempo, también hay quien lanza la mirada al futuro y no puede evitar pensar lo que posiblemente les espere con el paso de los años.

.....lo que predomina es cansancio de los profesionales aunque sorprendida por la calidad humana de los trabajadores en circunstancias especiales, fuera de ese momento existe cansancio, distanciamiento, segmentación de grupos que se unen por 
categorías profesionales y dentro de éstas por afinidades personales...... (Aux. Enfermería 2).

Es recurrente la manifestación respecto a que a las discrepancias entre los diferentes colectivos profesionales que comparten el trabajo, los problemas laborales se enconan y aparece el síndrome del profesional quemado también conocido como burnout que a grandes rasgos se puede definir como la exposición prolongada a factores estresantes que se traducen en estado de agotamiento psicológico, emocional y físico por la implicación en situaciones discordantes y problemáticas, si persiste se puede transforma en fatiga, ineficiencia, etc. desencadenando otros problemas que enmascaran la situación (Estévez Guerra, et al, 2002; García García, 2007; Simonelli Muñoz, et al, 2013).

Trabajar con personas mayores, quema mucho a la vez que hay que luchar contra el sistema, porque falta motivación, muchas personas no realizan el trabajo que deberían y no cumplen con sus función, pasando del trabajo y las personas responsables llega un momento que se cansan y entran en ese círculo vicioso y va creando apatía, desgana y malos rollos, se lucha contra corriente y se demandan equipos de trabajo que a nivel organizativo se niegan a que existan, considerando que se podrían ver amenazados los privilegios de algunos empleados de escalafones superiores, llámese personal facultativo, Administración, etc. (DUE, 1).

Es una idea generalizada que existen ciertos centros de trabajo con mayores problemas que otros debido a la gestión del equipo directivo y personal facultativo, que discrimina a otros colectivos, existiendo clasismo, no existiendo capacidad de comunicación descendente y evitando o poniendo freno a la comunicación ascendente debido a prepotencia e insolidaridad, lo que arrastra una serie de problemas que repercute en todo el sistema.

.(....).... Me gusta mucho mi trabajo aunque hay que reconocer que cansa mucho física y psicológicamente, este trabajo implica mucho sacrificio personal, además de por el trabajo en sí, por ser personas mayores algunas enfermas, requiere trabajar a turnos de mañana tarde y noche, festivos, etc., lo que crea muchísimo estrés especialmente desde los diplomados en enfermería debido a que existen situaciones límite que te obligan a tomar decisiones y de éstas depende la vida de una persona recayendo responsabilidades que en otros servicios no es así porque el médico está presente y dicha responsabilidad es suya. Los profesionales cuando se queman es porque les sobrepasan situaciones estresantes sin que se resuelven sus problemas, que en los centros de mayores hay muchos, desde los públicos a los privados. La comunicación entre trabajadores del mismo nivel y colectivo suele ser fluida pero entre los diferentes colectivos o es mínima o no existe, imponiéndose el ordeno y mando que no lleva a ningún sitio. Se echa en falta reuniones de trabajo interdisciplinares para resolver los problemas de trabajo para mejorar y que dicha mejora repercuta directamente en las personas mayores que viven en ella. La falta del trabajo en equipo en pleno siglo XXI es un fallo a nivel institucional inadmisible (DUE, 2).

La motivación junto a la comunicación contribuye a crear puente para que el trabajo sea más eficiente, eficaz y dinámico, siendo una idea generalizada que además de la dureza del trabajo con personas mayores se lucha contra el sistema que no facilita, sino que, más bien dificulta el trabajo impidiendo que exista coordinación interprofesionales de diferentes categorías que de una forma u otra están abocados a tener que trabajar juntos y prestar un 
servicio sanitario a personas mayores institucionalizadas en una residencia para mayores de titularidad en este caso publica.

Cuando existe discordancia profesional en las formas de actuar y realizar el trabajo, deben existir protocolos que unifiquen criterios, pero si no existen protocolos cada profesional actúa según su propio código de conducta, existiendo diversos y todos pueden ser buenos $\mathrm{y}$ efectivos pero discordantes lo que conlleva dificultades para poder trabajar y repercuten los problemas en el resto de los profesionales.

El trabajo físico es muy grande, lo que te va agotando con el paso de los años, todas comenzamos siendo muy jóvenes y el trabajo se superaba con las ganas de trabajar y la juventud, pero las cargas familiares y el transcurso del tiempo van minando día a día unido a las problemas de relación, problemas laborales sin resolver, las rencillas entre colectivos, la mala gestión administrativa, el deterioro de los usuarios, las demencias que es complicado con ellos. Existen otros centros que no diferencian entre profesionales, se trata a los residentes como personas respetando sus decisiones y actitudes de los facultativos, lo que no significa que no exista alguna jefatura que está ocupada por personas que quizás no deberían desempeñar ese trabajo por su falta de eficiencia................ (Aux. Enfermería 3).

La formación a lo largo de la vida se ha ido instaurando en las tres últimas décadas, se prevé que se cambiará de empleo u oficio varias veces en el transcurso de la vida laboral, por lo que hay que ir readaptándose a las nuevas realidades.

Desde hace unas décadas se pone en cuestión que los derechos sociales conseguidos a través de luchas sociales se podían perder, y se comienza a externalizar y subcontratar el trabajo, a flexibilizar las condiciones laborales, se crea inestabilidad lo que conlleva empeorar las condiciones de vida y una inestabilidad social que condiciona la calidad de vida, porque no existe protección social que asegure unas condiciones mínimas, la crisis económica con efecto dominó en el ámbito social ha puesto de relieve la inconsistencia de parte del sistema existente hasta el momento.

...Suscribir todas las circunstancias descritas por las anteriores compañeras, reiterando los esfuerzos físicos y la monotonía existentes en el centro sin perspectivas de cambio. Los cambios que se han producido en los últimos años lejos de mejorar lo que han producido ha sido deterioro, siendo uno de los mayores problemas la desmotivación del personal que desempeña sus funciones en el centro, por las diversas circunstancias que ya se han descrito. Las persecuciones de algunos empleados de categorías profesionales superiores sobre otros con menor nivel laboral y repercute directamente el trabajo y órdenes de trabajo sobre ellos, ha creado un ambiente enrarecido con acciones que han judicializado actuaciones y funciones laborales han deteriorado aun más el trabajo. Es cierto que todos tenemos derechos, y obligaciones, pero judicializando no se avanza, porque quien pierde judicialmente debe acatar sentencias y quien gana tampoco debe recrearse en la victoria, debiendo de resolver la situación para evitar enconamiento, fórmulas de arbitraje donde todos ganen y todos pierdan un poco pero viabilizando canales que permitan poder seguir trabajando y evitando sanciones que lleven a poner el centro de trabajo en el centro de mira de la Administración para que esta, la única fórmula que proponga sea el desmantelamiento del centro y la privatización de servicios amparándose en la inviabilidad de la existencia del centro. Todos estos problemas, el absentismo laboral aumenta, la 
gestión solo parchea, pero no entra en profundizar ni resolver el problema (Aux. Enfermería 4).

Es una idea generalizada que cada cierto tiempo y como máximo en 10 años debe existir un reciclaje profesional y rotación permitiendo y facilitando que los trabajadores puedan trasladarse a otros puestos, pero esto solo seria posible con acuerdos con el Servicio Andaluz de Salud, aunque esto nunca ha interesado a pesar de las propuestas que se han hecho por parte de los trabajadores a la Administración, sindicatos y Comité de Empresa, ha sido un tema tabú que mejor ni nombrar; lo que permite "no visualizar el problema y dejar hacer", hasta que el problema se ha hecho tan visible que lo que se vislumbra desde hace tiempo es que la Administración lo único que desea es enterrar el problema cerrando el centro que cause problemas, cuestión que se ha tratado en el Parlamento Andaluz, y ha tenido amplio eco en los medios de comunicación.

El principal problema de fondo es la falta de planificación y la estructura del trabajo, aunque es algo que podría constar en papel, no se lleva a la práctica ni se respeta por quienes deben planificar o tienen puestos de jefatura.

\section{A MODO DE CONCLUSIÓN}

Que la crisis actual va a marcar un antes y un después a todos los niveles desde sociedad, economía, empleo es un hecho indiscutible, pero ante grandes problemas también hay que buscar soluciones de consenso y las administraciones públicas deben tener un papel que impulse la recuperación económica y la creación de empleo, debiendo elaborar planes eficaces y efectivos de reactivación de empleo para impulsar la actividad económica, lo que no significa que se organicen criterios de eliminación de gastos innecesarios e introduzcan criterios de calidad, eficiencia, eficacia y efectividad desencadenando criterios de efectividad administrativa, evitando privatizaciones de servicios públicos amparándose en ineficacias: la Administración en las dos ultimas décadas ha construido centros residenciales para mayores pero ha externalizado su gestión, además de realizar mayor concierto de plazas de residencias privadas para personas dependientes desde la Administración pública, según su discurso para abaratar costes; esa disminución del gasto se traduce en precariedad laboral para sus empleados.

Cuando se habla de bienestar se vuelve la mirada al deporte y actividades físicas, pero sentirse bien pasa por otras cuestiones y también otros tiempos; y el trabajo ocupa una parte importante del tiempo, las inversiones para mejorar y que conduzcan a producir satisfacciones en los RRHH, también producirá ventajas en la empresa haciéndola mas efectiva y rentable, aunque la rentabilidad no siempre sea económica.

Independientemente de la tendencia ideológica que gobierne la comunidad Autónoma, las acciones son similares en la pésima gestión del empleo publico, aunque se mantiene el despilfarro en otros sectores.

Si las condiciones laborales empeoran día a día en general y de forma particular en centros para personas mayores dependientes ¿Qué pueden hacer los empleados de bajos niveles que dependen de la gestión de niveles supriores?; con las actuales coyunturas y circunstancias económicas bien poco o casi nada, pero no se puede entrar en apatía y defender el trabajo de atención publico, que permitirá mejores condiciones laborales para los trabajadores. 
En definitiva, el trabajo en residencias públicas de Andalucía se percibe como un problema de difícil solución para los profesionales que trabajan en ellas.

\section{BIBLIOGRAFÍA}

ALVES CORRÊA, D.; CIRERA OSWALDO, Y. y GIULIANI, A.C. (2013), "Vida con calidad y calidad de vida en el trabajo", Argentina, INVENIO. Revista de Investigación Académica, $\mathrm{n}^{\mathrm{o}} 16$ (30), pp. 145-163.

BELZUNEGUI, A.; PASTOR, I. (2011), "Género y pobreza, ¿feminización o socialización de la pobreza en España?", Barataria, Revista Castellano-Manchega de Ciencias Sociales, $\mathrm{n}^{\circ}$ 12, pp. 185199.

BOADA-GRAU, J.; COSTA-SOLÉ, J.; GIL-RIPOLL, C.: VIGIL-COLET, A. (2012), "Desarrollo y validación de dos escalas sobre las prácticas de retribución: PRG-13 y PRE-21", Psicothema, Vol. 24, no 3, pp. 461-469

CASTILLO MENDOZA, C.A. y GARCÍA LÓPEZ, J. (2001), "Marx, entre el trabajo y el empleo", Salamanca, VII Congreso Nacional de Sociología, 20 a 22 diciembre.

ESTÉVEZ GUERRA, G.J.; FARIÑA LÓPEZ, E.; MARRERO MEDINA, C.D.; NÚÑEZ GONZÁLEZ, E. y BRAVO MARTÍNEZ, J. (2002), "Síndrome de Burnout en una unidad de geriatría. Una aproximación a la realidad", Gerokrono. Revista de la sociedad española de Enfermería Geriátrica y Gerontológica, Vol. 13, $\mathrm{n}^{\circ}$ 3, pp. 137-142.

GARCÍA GARCÍA, J. M. (2007), Estudio sobre variables de personalidad y organizacionales que influyen en el síndrome de Bournout en los trabajadores de un Hospital Psiquiátrico Penitenciario, Sevilla, Universidad de Sevilla.

GASCO GARCÍA, E. (2011), "Derecho y trabajo asalariado: significado y tensiones. A propósito del libro de Antonio Baylos y Joaquín Pérez Rey, El despido o la violencia del poder privado", Barataria, Revista Castellano-Manchega de Ciencias Sociales no 12, pp. 173-183.

CSI-F (2013), “Administración, Sanidad y Empleo destruyeron 223.464 empleos en el último año", en prensa disponible en: http://www.csi-f.es/es/content/administracion-sanidad-y-empl

eo-destruyeron-223464-empleos-en-el-ultimo-ano, visitado: 25 de junio de 2013.

INSTITUTO NACIONAL DE ESTADÍSTICA (2013), http://ine.es/ss/Satellite?c=INEPublicacion_C $\&$ cid $=1259924856416 \&$ pagename $=$ ProductosYServicios\%2FPYSLayout \&L $=$ es_ES\&p $=12547351106$ 72\&param1=PYSDetalleGratuitas, visitado: 25 de junio de 2013.

LAVÍA, C.; OLAZARÁN, M.; ALBIZU, E. y OTERO, B. (2012), "Formación continúa en centros de FP y actividades de innovación en las pymes industriales", ARBOR, Ciencia, Pensamiento y Cultura, Vol. 188, pp. 153-170

SIMONELLI MUÑOZ, A. J; SOLANO GARCÍA, L. y CAMPILLO CANO, M. (2013), "Formación del personal de ayuda a domicilio y el beneficio generado en el usuario" Murcia. Enfermería Global, vol. $2, \mathrm{n}^{\circ} 30$, pp. 244-255.

SÁNCHEZ HERRERA, J. y PINTADO BLANCO, T. (2009), Imagen corporativa: Influencia en la gestión empresarial, Madrid, ESICA.

ZUBERO, I. (2006), "Las nuevas relaciones entre empleo e inclusión: flexibilización del trabajo y precarización vital”, Documentación social, n 143, pp. 11-30. 


\section{Breve currículo:}

\section{Concepción Nieto-Morales}

Doctora en Sociología. Acreditada como profesora contratada doctora. Evaluación Positiva de Docentia. Ha publicado varios libros como La violencia de género en el contexto de la globalización (2011) y ha actuado como coordinadora de otros como: El trabajo técnico en el ámbito judicial. Descendiendo a la práctica profesional (2012), Dykinson; La Violencia intrafamiliar: menores, jóvenes y género. Una mirada desde la práctica profesional, Bosch, (2012) o Los efectos del trabajo con personas mayores institucionalizadas, (2013), Dykinson.

\section{Lourdes Nieto-Cabrera.}

Diplomada Universitaria en Enfermería, Klinikum Darmstadt; Darmstadt, Alemania. Profesora de cursos de formación especializada. Coordinadora expertos universitarios: Intervención con familia, Infancia y Juventud; Intervención en casos de violencia, Coautora de varios artículos y capítulos de libros.

\section{Manuel Francisco Díaz González}

Licenciado en Sociología y Diplomado en Trabajo Social. Profesor Titular e investigador en el Departamento de Trabajo Social y SSSS en UPO. Desde 1985 a 1991, fue Subdirector del Área de Prácticas y miembro del Consejo de Dirección de la Escuela de Trabajo Social de Sevilla. Ha participado en programas europeos de Investigación desde 1996 hasta 2012. Coordinador académico movilidad programa Erasmus (movilidad de estudiantes y profesores), París y Amberes. 